\title{
JUURNAL.RU
}

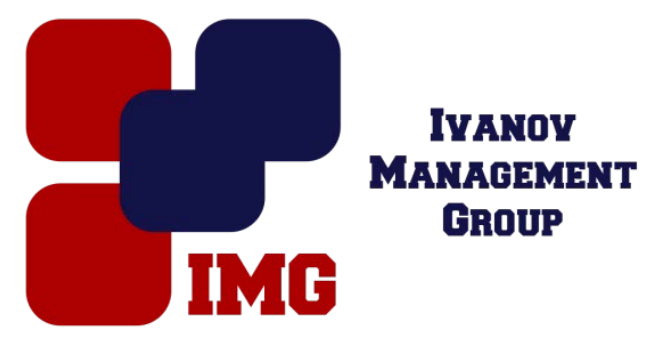

Котельников В.В.

ООО «Промышленная безопасность» Москва, Россия

doi: 10.18411/lj-31-10-2016-2-06

idsp 000001:lj-31-10-2016-2-06

\section{Алгоритм применения системы надежности (с ответной реакцией)}

Метод прогнозирования предельного ресурса и надежности функционирования элементов и конструкций из полимерных композиционных материалов на основе перспективных методик (включающих элементы искусственного интеллекта на базе искусственной нейронной сети) и хронологического анализа результатов факторов производства изделий, их эксплуатации, диагностики и контроля, основывается на прогностическом моделировании.

Прогностическая модель предельного ресурса эксплуатации сложных технических систем базируется на функции определения надежности технической системы. Учитывая многокомпонентность технической системы и динамическую природу внутренних и внешних факторов надежность можно определить как возможность объекта (производства, организации, сообщества, региона) прогнозировать, сохранять устойчивость, поглощать воздействия, реагировать, адаптироваться и восстанавливаться при нежелательном воздействии.

Поскольку надежность критически важной инфраструктуры важна для обеспечения собственной работоспособности и от нее зависит общественные/региональные процессы, в связи с этим можно степенью надежности выразить степень безопасности сложных технических систем и приравнять эти понятия для научно-технических задач, связанных с сложными 
техническими и информационными системами в практическом поле рассмотрения.

Метод оценки безопасности на уровне объекта основывается на определении надежности объекта путем сбора информации о четырех укрупненных группах мер повышения надежности: обеспечение готовности, меры по смягчению последствий, возможности реагирования и механизмы восстановления [1,2]. В таблице 1 представлены связи шести компонентов надежности с процессами, обеспечивающими надежность объекта. Эти связи обеспечивают основу для разработки опросника, который будет использоваться для выявления необходимой информации для оценки надежности на уровне объекта.

Таблица 1

связи между компонентами надежности и прочессами, повышающими надежность

\begin{tabular}{|c|c|c|c|c|c|}
\hline Прогнозирование & Устойчивость & Поглощение & Реагирование & Адаптация & Восстановление \\
\hline Готовность & \multicolumn{2}{|c|}{ Смягчение последствий } & \multicolumn{2}{|c|}{ Реакция } & Восстановление \\
\hline $\begin{array}{c}\text { Действия по } \\
\text { определению } \\
\text { наличия угроз в } \\
\text { среде, где } \\
\text { находится объект }\end{array}$ & \multicolumn{2}{|c|}{$\begin{array}{c}\text { Действия по уменьшению } \\
\text { опасности последствий } \\
\text { угрозы }\end{array}$} & \multicolumn{2}{|c|}{$\begin{array}{c}\text { Непосредственные и } \\
\text { постоянно выполняемые } \\
\text { процессы в ответ на } \\
\text { побочные эффекты } \\
\text { нежелательного события }\end{array}$} & $\begin{array}{c}\text { Процессы, } \\
\text { направленные на } \\
\text { эффективный } \\
\text { возврат состояния } \\
\text { объекта к } \\
\text { допустимому } \\
\text { уровню }\end{array}$ \\
\hline
\end{tabular}

Региональная/общественная безопасность зависит от надежности всех подсистем, в том числе надежность критически важной инфраструктуры, экономики, гражданского общества, государственной власти (в том числе оперативных служб) и других элементов. С увеличением количества и сложности этих элементов будет увеличиваться и число проблем, которые необходимо разрешить для оценки надежности, поскольку осуществляется переход с оценки надежности на уровне объекта до уровня региона/общества, где критически важная инфраструктура всего лишь один из компонентов. Такие проблемы включают в себя неопределенность взаимосвязей (например, состав конкретных цепочек событий), отсутствие данных, ограниченное время и бюджет, которые не позволяют организовать сбор всей необходимой информации для комплексной оценки региональной/общественной безопасности. По крайней мере частично эти проблемы могут быть разрешены 
путем применения «системного подхода» к оценке надежности. В этом случае можно производить оценку надежности отдельных подсистем на разных уровнях. При рассмотрении систем более высокого уровня можно определить наиболее важные для оценки надежности системы низкого уровня. В свою очередь, по отношению к системам более низкого уровня, определяются наиболее важные компоненты из числа тех, информация о которых имеется [3].

Определим надежность элементов сложной технической системы как возможность их восстановления, или другими словами возможность произвести «возврат в рабочее состояние» (устойчивость) при наступлении неблагоприятных последствий, вызванных естественным или человеческим фактором. На рисунке 1 представлен алгоритм работы метода.

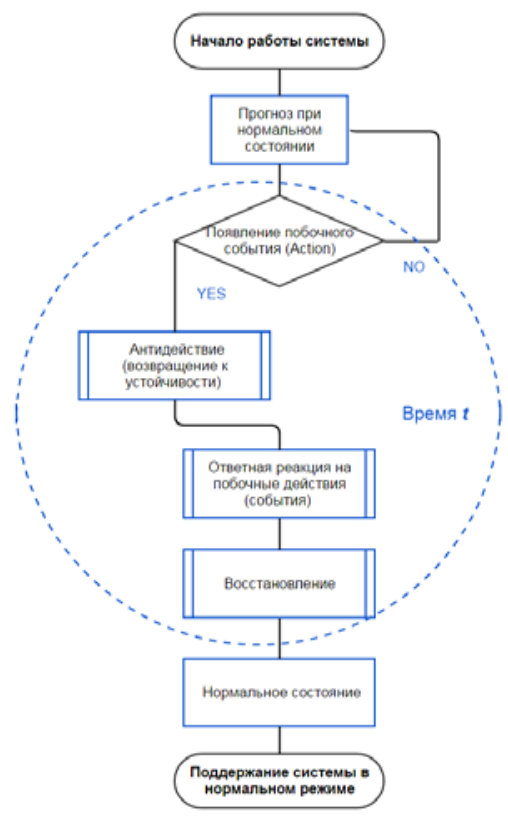

Рисунок 1. Алгоритм применения системы надежности (с ответной реакцией)

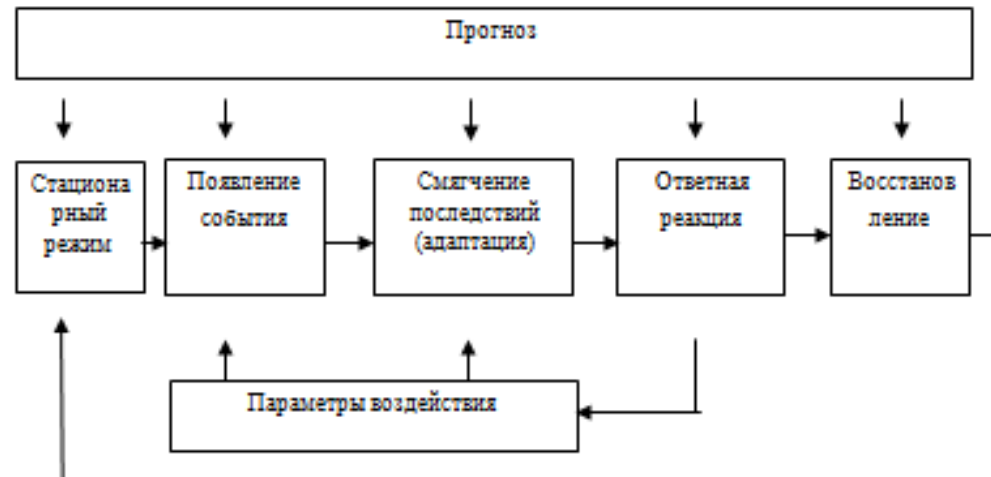

Рисунок 2 Функциональная схема способа обеспечения безопасности сложных технических систем. 
Прогноз состояния конструкции можно отнести к вероятностной модели, при этом сами характеристики состояния конструкции можно выразить в элементах теории нечетких множеств.

Уровней дефектности.

Таблица 1

\begin{tabular}{|c|c|c|}
\hline $\begin{array}{c}\text { Уровень } \\
\text { дефектности }\end{array}$ & Характеристика дефектов & $\begin{array}{c}\text { Модификатор нечеткого } \\
\text { множества }\end{array}$ \\
\hline I & $\begin{array}{c}\text { Механические повреждения отсутствуют, деформаций и } \\
\text { перемещений, слабые проявления коррозионных } \\
\text { воздействий, без снижения несущей способности } \\
\text { материалов }\end{array}$ & «слабый» \\
\hline II & $\begin{array}{l}\text { Наличие небольших механических и коррозионных } \\
\text { повреждений, присутствие некритических деформаций }\end{array}$ & «умеренный» \\
\hline III & $\begin{array}{c}\text { дефекты, которые оказывают частичное влияние на } \\
\text { дальнейшую эксплуатацию конструкций, мелкие трещины, } \\
\text { деформации и снижение прочностных показателей } \\
\text { материалов в пределах, регламентированных нормативно- } \\
\text { технической документацией }\end{array}$ & «значительный» \\
\hline IV & $\begin{array}{c}\text { Опасные дефекты не приводящие к разрушению элементов, } \\
\text { но ограничивающие дальнейшую эксплуатацию } \\
\text { конструкций - значительные механические и коррозионные } \\
\text { повреждения, сверхнормативные деформации и снижение } \\
\text { прочностных показателей материалов, что требует } \\
\text { проведения мониторинга динамики развития дефектов } \\
\text { и/или выполнения корректирующих мероприятий (ремонт, } \\
\text { снижение нагрузок, реконструкция и т.д.). }\end{array}$ & «предельный» \\
\hline
\end{tabular}

При измерительной оценке выставляются определенные модификаторы по таблице 1:

- уровень механических повреждений;

- уровень коррозионных повреждений;

- уровень деформаций и перемещений;

- уровень снижения несущей способности материала;

- количество элементов, имеющих дефекты и повреждения;

- общее количество элементов.

Уровень повреждений и деформаций, при исследовании конструкций сложной формы, состоящей из множества узлов, имеет плохую степень формализации. При реальном обследовании измерения делаются только на части конструкции, поэтому определить причины достаточно сложно, однако при использовании аппарата абдуктивного логического вывода это представляется возможным $[4,5]$. 
В случае применения логических подходов в качестве программного средства диагностики, часть знаний должна использоваться для рассуждений, обеспечивающих объяснения выведенных заключений. Но традиционная логика имеет свои ограничения, особенно в условиях неполной или неопределенной информации. В этом случае решением задачи становится выявление и установление причинно-следственных связей. В подобных ситуациях применение таких логических выводов, как индуктивные, дедуктивные и выводы по аналогии, невозможно, т.к. для своей работы они требуют наличия всей информации о диагностируемой системе.

Поэтому, при решении задачи выявления и установления причинноследственных связей, следует использовать абдуктивный [6] вывод для объяснения наблюдаемых (или установленных) фактов.

Таким образом, задача прогнозирования предельного ресурса эксплуатации может быть сформулирована как задача аппроксимации функции многих переменных. Необходимо построить некоторое отображение $\mathrm{H} \rightarrow \mathrm{Y}$ такое, чтобы на каждый возможный входной образ, представленный вектором входных данных (Н) формировался правильный выходной вектор прогноза (Y).

Использование искусственных нейронных сетей (ИНС) в качестве аппарата для установления зависимости между входными данными и результатами прогноза обусловлено способностями сети к параллельной и распределенной обработки информации, что позволяет значительно увеличить скорость работы алгоритма, внести элементы искусственного интеллекта и повысить эффективность прогноза. При этом нейросетевой метод моделирования не требует априорного задания вида исследуемой зависимости.

С помощью данного метода существует возможность прогнозирования предельного ресурса и надежности функционирования элементов и конструкций, в т.ч., из полимерных композиционных материалов на основе перспективных методик (включающих элементы искусственного интеллекта на базе ИНС) и анализа результатов испытаний, диагностики и неразрушающего контроля с использование абдуктивного методов:

1. Новая методология создания научно-методических основ и многоканальных многопараметровых программно-аппаратных 
средств новых технологий оценки безопасности любой сложной технической системы.

2. Прогностические модели и программно-аппаратные средства с использованием элементов искусственного интеллекта оценки предельного ресурса эксплуатации элементов и систем и конструкций.

3. Методику прогнозирования предельного ресурса эксплуатации элементов и конструкций из полимерных композиционных материалов в процессе испытаний, хранения и эксплуатации.

4. Новую сенсорную многоячеистую систему мониторинга объектов и методологию ее использования. 


\section{Литература}

1. Коршаков А.В. Обзор некоторых методов реконструкции томографических изображений. //Контроль. Диагностика. - М.: Машиностроение.-№3(105) 2007.-с. 45-49

2. Бейтс Р., Мак-Доннелл М. Восстановление и реконструкция изображений: Пер. с англ. - М.: Мир, 1989. - 336 с.

3. Чичигин Б.А., Кеткович А.А., Чернов Л.А. Современные лазерные системы контроля геометрии сложных поверхностей //Тезисы докладов семнадцатой российской НТК с международным участием «Неразрушающий контроль и диагностка», Екатеринбург, ИМАШ, УрО PAH, 2005-c. 168.

4. Будадин О.Н., Бекаревич А.А., Морозова Т.Ю. Математическое моделирование процесса теплового контроля локальных дефектов металлических изделий сложной формы на примере лопаток турбин газотрубных двигателей В мире научных открытий. №8(68), Красноярск, 2015 , с. $34-43$.

5. Будадин О.Н., Бекаревич А.А., Морозова Т.Ю. Тепловой автоматизированный контроль качества и диагностики технического состояния лопаток турбин газотурбинных агрегатов с оценкой надежности эксплуатации. М.: Спектр, 2014, 382 с. 